\title{
Nucleoid Structure of Escherichia coli as Revealed by Scanning Transmission Electron Microscopy (STEM) and by 3D-Reconstruction of Z-contrast Images of Serial Sections
}

\author{
Bleher Reiner ${ }^{1,2,3}$, Ying Guo ${ }^{1,2}$, Eric W. Roth ${ }^{1,2}$, Nastaran H Yazdi ${ }^{4}$, Reid C. Johnson ${ }^{5}$, Calin Guet ${ }^{6}$, John \\ Marko $^{4,7}$, and Vinayak P Dravid ${ }^{1,2}$ \\ 1. Dept. of Materials Science and Engineering, Northwestern University, Evanston, IL 60208, USA \\ 2. NUANCE Center, Northwestern University, Evanston, IL 60208 \\ 3. Chemistry of Life Processes Institute, Northwestern University, Evanston, IL 60208, USA \\ 4. Department of Physics \& Astronomy, Northwestern University, Evanston, IL 60208, USA \\ 5. Department of Biological Chemistry, David Geffen School of Medicine, University of California at \\ Los Angeles, CA 90095, USA \\ 6. IST Austria, A-3400 Klosterneuburg, Austria \\ 7. Department of Molecular Biosciences, Northwestern University, Evanston, IL 60208, USA
}

The nucleoid is the compartment in bacteria that contains the DNA. Its structure has been examined extensively with fluorescence- and conventional light microscopy. It has been shown that in rapidly growing bacteria the nucleoid shows a more dispersed structure compared to a more compact structure of the nucleoid under low-growth conditions. Higher spatial resolution studies with TEM (Transmission Electron Microscopy) on resin embedded or cryo-processed samples have delivered ambiguous results for the overall shape of the nucleoid, and for the appearance of the chromatin inside the nucleoid, when different fixation and contrasting protocols were used $[1,2]$. With our protocol, the nucleoid resembles a space with lesser density than the surrounding cytoplasm; it contains fibrillar material and extends along the longitudinal axis of the bacterium. This is corroborating results obtained with osmium tetroxide fixation of E. coli under R-K conditions, and observations of high-pressure frozen and freeze substituted samples [2].

In this study, we reconstructed the nucleoid shape in serial sections of resin embedded E. coli cells that were cultured in media for rapid-, and for slow growth conditions. The reconstructed shapes of the nucleoids showed pronounced differences. In rapid-growth medium, the nucleoid shape was irregular and dispersed while in slow-growth medium, the nucleoid appeared more compact and regular. A comparable change of nucleoid appearance has been shown in vitreous sections of exponentially growing- and stationary phase cells of $D$. radiodurans [3]. Also, the results shown are in agreement with findings of a recent study of E. coli cells by in vivo fluorescence [4].

The results confirm that the shape of the nucleoid of $E$. coli is affected by the growth conditions the bacteria are exposed to, and that these changes can be observed in chemically fixed and resin embedded samples of the bacteria.

E. coli cells were grown at $37^{\circ} \mathrm{C}$ in in LB medium (fast growth) or in M9 medium (slow growth). For resin embedding, the cells were fixed by adding formaldehyde and glutaraldehyde to a final concentration of $4 \%$ and $0.1 \%$, respectively, to the bacterial suspension. The specimens were postfixed with $2 \%$ osmium tetroxide and dehydrated in an ascending series of ethanol. The $50 \%$ ethanol solution contained $1 \%$ uranyl acetate. The samples were infiltrated with LR-White resin and polymerized. Ultrathin serial sections of $60 \mathrm{~nm}$ thickness were collected on Formvar/carbon coated slot grids. Z-contrast images were taken without further contrasting with a STEM (Hitachi HD2300) at 200kV acceleration voltage. Image stacks of 9 sections (slow growth, M9 medium samples) and of 13 sections (fast growth, LB medium samples) were aligned and processed with the Fiji software package [5]. 
References:

[1] Eltsov, M. and B. Zuber, Journal of Structural Biology, 156(2) (2006) p. 246-254.

[2] Pilhofer, M., et al., in "Chapter 2 - Bacterial TEM: New Insights from Cryo-Microscopy", in Methods Cell Biol, M.-R. Thomas, Editor, Academic Press, (2010) p. 21-45.

[3] Eltsov, M. and J. Dubochet, Journal of Bacteriology, 187(23) 2005 p. 8047-8054.

[4] Yazdi, N.H., et al., Molecular Microbiology,. 86(6) 2012 p. 1318-1333.

[5] Schindelin, J., et al., Nat Meth, 9(7) 2012p. 676-682.
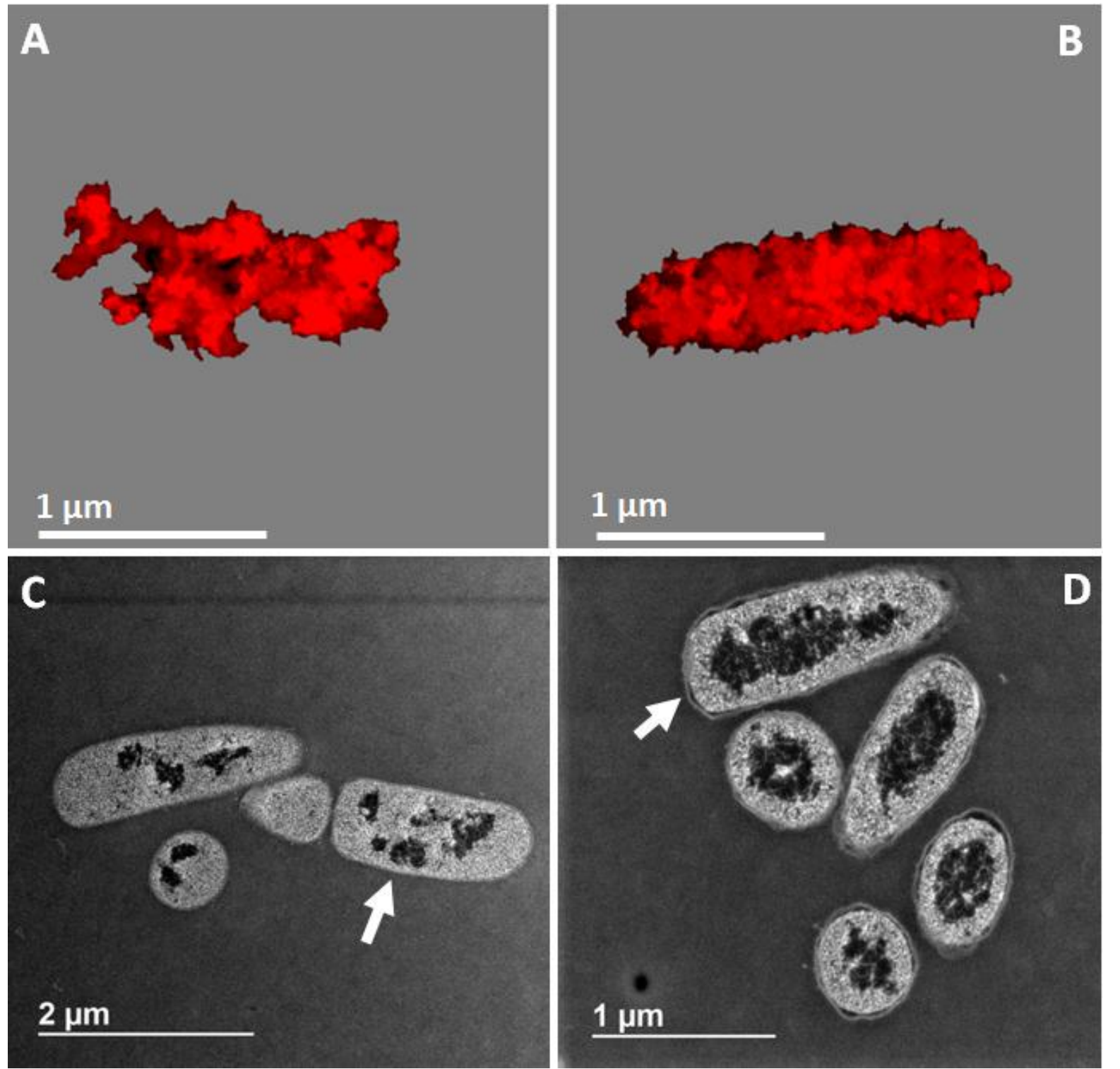

Figure 1. Reconstructed nucleoids (upper panel) and one Z-contrast image from each corresponding image stack of serial sections (bottom panel) of E. coli cultivated in fast-growth LB medium (A, C), and in slow-growth M9 medium (B, D). Arrows depict the cells chosen for reconstruction. 